\title{
Redes sociales y estudiantes: motivos de uso y gratificaciones. Evidencias para el aprendizaje
}

\author{
Rosa García-Ruiz (*), Ramón Tirado Morueta $\left(^{* *}\right)$ y Ángel Hernando Gómez ${ }^{*}$ ) \\ (*) Universidad de Cantabria - España (**) Universidad de Huelva - España
}

\begin{abstract}
RESUMEN
Aunque las nuevas generaciones utilizan las redes sociales como un medio de comunicación habitual, en el ámbito educativo aún no se han utilizado de manera generalizada por los docentes como recurso didáctico. Conocer las gratificaciones que encuentran las nuevas generaciones al usar las redes sociales, permitirá al profesorado hacer un uso estimulante de estos medios en la educación secundaria y universitaria. El objetivo de este trabajo es conocer cuáles son las gratificaciones que encuentran los estudiantes de educación secundaria y universitaria al utilizar intensamente las redes sociales Facebook, Instagram y YouTube. Utilizando la teoría de Usos y Gratificaciones, se recogen datos a través de un cuestionario de estudiantes españoles de educación secundaria (N=260) y universitarios $(\mathrm{N}=315)$. Los resultados muestran que los estudiantes de secundaria buscan una mayor presencia social y velan por su propia imagen, mientras que los estudiantes universitarios buscan entretenimiento. Finalmente, se discuten los resultados y las implicaciones para un uso estimulante y didáctico de las redes sociales en contexto de enseñanza-aprendizaje.
\end{abstract}

Palabras Clave: estudiantes, usos y gratificaciones, redes sociales, generación Z, generación Y.

\section{Social media and students: Motivs of use and gratifications. Evidences for learning}

\section{ABSTRACT}

Even though new generations use social media as a common media, their use in the educational area hasn't still been generalized among teachers as a didactic resource. Knowing the gratifications that new generations find when using social networks will allow teachers to use an stimulating use of this media in High School and University education. The purpose of this paper is to know which are the gratifications that High School and University students get from using intensely the social media: Facebook, Instagram and YouTube. Using the theory of Uses and Gratifications, data is collected through a survey of Spanish students from Secondary Education $(\mathrm{N}=260)$ and University $(\mathrm{N}=315)$. The results show that High School students look for a higher social presence and look after her own image, while University students look for entertaining. Finally, the results and the implications for an stimulating and educational use of the social media in the context of teaching-learning are discussed.

Keywords: students, use and gratifications, social networks, Z generation, Y generation.

\section{Introducción}

Los sitios de redes sociales (SRS), tal y como plantea $\underline{\text { Xie }}$ (2014), están adquiriendo cada vez más relevancia en la vida de los adolescentes y jóvenes, siendo las actividades online muy frecuentes para estos usuarios desde edades cada vez más tempranas (Marsh, 2014). El acceso a internet, la disposición personal de smartphones y la posibilidad de descargar aplicaciones generan la proliferación de perfiles en redes sociales como Facebook, YouTube o Instagram, siendo consideradas por los más jóvenes como un medio de socialización que les permite estar conectados y mostrarse en la red (Almansa-Martínez, Fonseca, Castillo-Esparcia, 2013).
Dichas experiencias de socialización a través de las redes sociales están en gran parte mediadas por el intercambio de imágenes, lo que promueve una cultura hacia lo visual y lo que contribuye a la transformación en la manera de relacionarse, así como en la percepción y la construcción de la identidad personal y el sentido del yo (Svensson \& Russmann, 2017). Los menores y jóvenes han hecho ostensible históricamente la necesidad de expresarse y de participar en sociedad encontrando en las redes sociales la posibilidad de manifestar sus preocupaciones, pensamientos, sentimientos e intereses (Herrero-Diz, Ramos-Serrano \& Nó, 2016).

De este modo y a pesar de que la utilización de redes sociales por parte de los jóvenes puede suponer ciertos riesgos 
como, por ejemplo, adicción, acceso a contenidos inapropiados o ciberbullying, también puede implicar una gran variedad de posibilidades para promover su bienestar (Burbules, 2016). En este sentido, diversos estudios ponen de manifiesto las ventajas y oportunidades del uso de redes sociales en relación con su potencial educativo (Tur, Marín-Juarros \& Carpenter, 2017), así como con la mejora del rendimiento académico de los estudiantes (Barajas Meneses \& Álvarez Morán, 2013; González, Lleixà \& Espuny, 2016). Otras investigaciones comprueban el uso concreto de ciertas redes sociales en el ámbito educativo, obteniendo resultados que indican que se incrementa y facilita la interacción entre los jóvenes universitarios mediante la utilización de Whatsapp (Andújar-Vaca \& Cruz-Martínez, 2017; Yeboah \& Ewur, 2014); que existe una mejora en los procesos comunicativos entre los estudiantes y el profesor a través de Facebook (Herhkovizt \& Forkosh-Baruchel, 2017); que el aprovechamiento de YouTube puede suponer un escenario para aumentar la participación y expresión de los usuarios (Yarosh, Bonsignore, McRoberts \& Peyton, 2016); o que Instagram es una aplicación que ofrece la posibilidad de mostrar la creatividad (Dumas, Maxwell-Smith, Davis, \& Giuletti, 2017).

En este contexto en el que se evidencia el potencial educativo de los SRS como recursos para favorecer el aprendizaje significativo y mejorar las relaciones interpersonales entre los miembros de la comunidad educativa, surge el propósito de este estudio que pretende comprender cuáles son las motivaciones que estimulan a los adolescentes y jóvenes a utilizar algunas de las redes sociales de carácter genérico más populares, como Facebook, YouTube o Instagram y comprobar en qué medida la edad y la pertenencia a una generación influye en la relación entre las gratificaciones y la intensidad de uso de estas redes sociales. Para este fin, la investigación se basará en la teoría de los Usos y Gratificaciones (UyG), por ser una de las más utilizadas y reconocidas. Este enfoque teórico sostiene que la utilización de un medio particular es motivada por las gratificaciones que las personas aspiran a encontrar haciendo uso del mismo, por tanto, tiene sentido útil y está orientada hacia la consecución de una meta y la obtención de satisfacción (Palmgreen \& Rayburn, 1979). Asimismo, cabe destacar que las condiciones históricas, sociales y tecnológicas ejercen influencia en el uso de los medios y en sus efectos (Leung, 2013), por lo que la identidad o pertenencia generacional (Zhitomirsky-Geffet \& Blau, 2016) o el sexo (Oberts, Chamarro, \& Renau, 2016), pueden determinar las gratificaciones que un usuario obtenga y valore en el uso de un medio. A partir de los resultados de esta investigación se pretende contribuir empíricamente al conocimiento científico sobre el uso que hacen las generaciones jóvenes de las redes sociales y fomentar la comprensión de los educadores acerca de las posibilidades y beneficios derivados de la utilización de estos recursos de comunicación social en el ámbito académico, con el fin de adecuar las prácticas educativas a las necesidades e intereses de los estudiantes y de reconsiderar las formas y estilos de enseñanza que permitan la superación del currículum tradicional (Gerják, 2016).

Para alcanzar este objetivo, se han llevado a cabo dos estudios trans-generacionales. El primero con estudiantes pertenecientes a la generación Z (Schroer, 2008), concretamente con alumnos adolescentes de educación secundaria con edades comprendidas entre los 13 y los 18 años y el segundo con alumnado perteneciente a la generación Y (Coombes, 2009) o Millenials (Howe \& Strauss, 2000), específicamente con jóvenes que realizan estudios universitarios con edades que oscilan entre los 19 y los 38 años.

\section{Uso de internet y sitios de redes sociales (SRS) en el entorno} multipantalla

La utilización de medios online y sitios de redes sociales (SRS) ha ido adquiriendo una importancia cada vez mayor en la última década y se ha convertido en uno de los hábitos de comportamiento más extendidos entre la ciudadanía, debido a la ubicuidad y la convergencia de los dispositivos en el entorno multipantalla (Parra Castrillón, 2010; Sabater, Martínez, \& Santiago, 2017).

Asimismo, conviene mencionar que una gran parte de los usuarios de estas redes sociales son adolescentes y jóvenes, por lo que se hace necesaria una alfabetización en este ámbito que les permita valorar las posibilidades y riesgos derivados de su utilización y lograr el equilibrio adecuado entre las actividades de intercambio de contenido e información, la importancia de la privacidad y la necesidad de respetar la propiedad intelectual de los demás internautas (Gathegi, 2014). En esta investigación se han seleccionado, como objeto de estudio, tres redes sociales muy populares entre estudiantes de secundaria y universitarios: Facebook, Instagram y YouTube.

En primer lugar, cabe destacar que Facebook es una de las redes sociales por excelencia preferida por adolescentes, jóvenes y adultos, constituyéndose como una institución social que sirve para compartir contenidos, conectarse con otros usuarios y como plataforma de exploración y divulgación de información sobre la identidad personal (Jordán-Conde, Mennecke \& Townsend, 2014). Esta plataforma ofrece funcionalidades que permiten crear e interaccionar con una comunidad virtual de amigos a través de solicitudes de amistad, actualizaciones de estado, publicación de imágenes y vídeos e interactividad con otros usuarios mediante etiquetas, reacciones a las publicaciones o comentarios.

Por otra parte, Instagram surge en el año 2010 como una aplicación para el uso en teléfonos móviles y rápidamente se populariza especialmente entre los más jóvenes como una red social en la que los usuarios pueden subir y compartir fotos y vídeos (Hu, Manikonda, \& Kambhampati, 2014). El usuario tiene a su disponibilidad herramientas para editar las fotografías, como filtros, además de poder añadir la ubicación y etiquetar a otras personas con las que comparte la red. Actualmente permite también enviar mensajes o publicaciones de manera directa a otros usuarios, grabar y subir vídeos de un minuto, publicar varias fotografías en un mismo post y compartir las publicaciones en otras redes sociales. Asimismo, permite interactuar en las fotografías y vídeos de otros usuarios, señalando "me gusta" y añadiendo comentarios. En cuanto a las opciones de privacidad del perfil, esta aplicación ofrece la posibilidad de configurar la cuenta como pública o privada. En esta última opción el usuario podrá decidir qué solicitudes de amistad acepta y qué usuarios pueden visualizar su perfil. En 2016, Instagram añadió la posibilidad de publicar fotos y vídeos de 15 segundos de duración en lo que se denomina "Instagram Stories", una función al estilo de Snapchat, en la que las publicaciones desaparecen de la red en un día y los usuarios pueden visualizar quién ha visto su contenido. En sus últimas actualizaciones, la aplicación ha incorporado la opción de añadir máscaras, encuestas y gifs a estas publicaciones e incluso archivarlas en el perfil del usuario para que no desaparezcan en 24 horas. Por último, también cabe destacar que a través de esta aplicación se puede transmitir en directo, funcionalidad parecida a la ofrecida por Periscope o Facebook Live, con la opción de guardar el vídeo durante un día. Como nueva funcionalidad se ha añadido recientemente la posibilidad de compartir el directo con otro usuario, lo que supone una novedosa y original forma de interacción entre otros miembros de esta comunidad. 
Por último, YouTube, creado en 2005 como un repositorio de vídeos en la nube, es considerado una red social porque permite interactuar a los usuarios, compartiendo vídeos y comentarios, llegando a convertirse en el tercer sitio web más visitado y su buscador el segundo más usado después de Google. YouTube ofrece la posibilidad de ver vídeos, crearlos y compartirlos, de votar vídeos con "me gusta" o "no me gusta". Una novedad de esta red es la posibilidad de crear un canal propio, en el que el usuario puede archivar sus vídeos favoritos, creando secciones y listas de reproducción, además de seguir otros canales. Youtube se ha hecho también enormemente popular por los denominados "YouTubers", que se están convirtiendo en un fenómeno de masas, con gran influencia especialmente entre los más jóvenes. YouTube, a diferencia de otras redes sociales permite obtener ganancias económicas a partir de un número mínimo de seguidores a un canal y de visualizaciones a cada vídeo, vinculadas generalmente a la negociación con marcas publicitarias patrocinadoras (Smith, Fischer y Yongiian, 2012).

\section{La teoría de Usos y Gratificaciones (UyG)}

La teoría de Usos y Gratificaciones (UyG) sostiene que los individuos realizan una selección de manera activa de los medios de comunicación en función de sus intereses y necesidades, con la finalidad de satisfacer sus deseos y obtener una recompensa (Katz, Blumer \& Gurevitch, 1974). A partir de este enfoque se puede comprobar cuáles son las motivaciones y las razones que impulsan a los usuarios a seleccionar un determinado medio o, en este caso, sitio de red social.

En este sentido, según la teoría de UyG, la búsqueda de sociabilidad, información y entretenimiento son los principales motivos por los que los jóvenes tienen una alta implicación en la comunicación social digital y por el que otorgan un gran valor a las posibilidades de participación e información que les ofrecen las redes, así como por la obtención de beneficio social que les permite el contacto con otros usuarios (García Galera \& Fernández Muñoz, 2016). Del mismo modo, la curiosidad, la popularidad, la posibilidad de mantener contacto con amigos y la oportunidad de establecer nuevas relaciones o recuperar antiguas amistades se establecen como motivaciones que conllevan al uso de las redes sociales por parte de los jóvenes (Urista, Dong \& Day, 2009). Por su parte, Vasanth y Swamy (2013) destacan que el acceso a la comunicación con grandes grupos de personas se produce con relativa facilidad y rapidez, lo que supone un impacto que influye en la dinámica social, reduciendo las inhibiciones sociales y provocando nuevas acciones normativas. Por tanto, la valoración de los SRS que realizan los jóvenes es positiva en relación con las funcionalidades y posibilidades que ofrecen este tipo de servicios por su carácter gratuito, por la comodidad y utilidad para establecer relaciones con amigos y conocidos, por ser un medio para la socialización, expresión y el entretenimiento, así como por la facilidad para compartir contenidos, informarse y conseguir popularidad (Igartua \& Rodríguez-de-Dios, 2016; Fondevila-Gascon, Mir, Aguinaga, Carreras, \& Del Olmo 2014; Rubio-Romero \& Perlado Lamo de Espinosa, 2017). Del mismo modo, la utilización de redes sociales y el establecimiento de conversaciones personales a través de dispositivos móviles, además de fortalecer las relaciones personales de los adolescentes con familiares y amigos, permite la promoción de su compromiso cívico (Xie, 2014), así como el empoderamiento y la participación juvenil (Fuentes, García, \& Aranda, 2017).

\section{Adolescentes y jóvenes ante los medios. Análisis generacional}

Diferentes terminologías han sido empleadas para referirse a los menores y jóvenes en función de sus características o las generaciones a la que pertenecen, entre los que se podrían destacar, tal y como plantean Herrero-Diz, Ramos-Serrano y Nó (2016), conceptos como Millenials, nativos digitales, Generación Y, Generación Z, Generación Google, Generación interactiva, App Generation o Generación Selfie, cuyo nexo de unión es su afición e interés por la tecnología y su utilización en el desarrollo de su cotidianeidad (Pérez-Escoda, Castro-Zubizarreta \& Fandos-Igado, 2016). En este sentido, a pesar de las diferentes denominaciones que se empleen para hacer referencia a los jóvenes, todas las acepciones muestran algunas características en común que definen a este sector poblacional como, por ejemplo, la capacidad multitarea, la necesidad de comunicarse y estar conectado, la importancia de la inmediatez, la creación de contenidos, la familiaridad que muestran ante los formatos audiovisuales y el uso de dispositivos, así como la habilidad colaborativa y la capacidad de producir y consumir contenidos incluso de manera simultánea (Herrero-Diz, Ramos-Serrano y Nó, 2016).

Así, la Generación Y hace referencia a aquellos adultos jóvenes nacidos entre 1977 y 1994, conocidos también como Millenials o segunda generación "Baby Boomers", mientras que la Generación $\mathrm{Z}$ engloba a los niños o adolescentes que nacieron entre 1995 y 2012, por lo que ambas generaciones forman parte hoy en día del sistema educativo, ya sea en las etapas de educación primaria y secundaria o en el ámbito universitario o de formación profesional (Fernández-Cruz y Fernández-Díaz, 2016). Expertos en educación mediática insisten en la necesaria incorporación de la educación en medios en el currículum académico, entendiendo la alfabetización mediática como una apuesta para promover tanto la recepción como la producción de mensajes y contenidos de manera crítica, creativa, responsable y emocional y de alcanzar una sociedad prosumidora (García-Ruiz, Ramírez-García, \& Rodríguez-Rosell, 2014).

En relación al uso de redes sociales por parte de los jóvenes y adolescentes, es preciso señalar que existe un amplio abanico de posibilidades, usos y motivaciones que impulsan a este sector de la población a la utilización de este tipo de recursos. De este modo, de acuerdo con Israel (2017), las redes sociales son populares entre los niños y jóvenes, ya que conciben los SRS como plataformas naturales en su cotidianeidad y en su vida social y les permiten estar conectados con una gran cantidad de amigos, interaccionar con usuarios de distintas procedencias y culturas y derribar barreras que se dan en la comunicación cara a cara como la controlabilidad de autopresentación o la superación de cuestiones espacio temporales. En el ámbito académico, estudios como el realizado por Hershkovizt y Forkosh-Baruch (2017) ponen de manifiesto que en la actualidad los procesos comunicativos entre profesorado y alumnado se realizan en gran medida a través de redes sociales, constituyéndose como recursos facilitadores de las relaciones y los procesos de enseñanza-aprendizaje.

Por tanto, mediante este estudio se pretende comprender el uso que hacen las generaciones jóvenes de algunas de las redes sociales más utilizadas (Facebook, Instagram y YouTube), así como las gratificaciones y motivaciones que definen el comportamiento de los adolescentes y jóvenes en los SRS, cubriendo la falta de investigaciones transgeneracionales realizadas en este ámbito (Manzi, Coen, Regalia, Yevenes, Giuliani \& Vignoles, 2018). De este modo, se trata de ofrecer a los educadores y gestores educacionales un mayor conocimiento sobre las tendencias de uso de redes sociales por parte de los jóvenes, con el fin de contribuir con aportaciones que pudieran servir para que se incorpo- 
ren estos medios de comunicación social de manera más práctica y eficaz en los procesos de enseñanza-aprendizaje, adaptando de este modo las experiencias educativas a las características e intereses del alumnado. Para lograrlo se han planteado dos preguntas de investigación:

PI1 ¿Existen diferencias significativas en la frecuencia de uso de la redes sociales (Facebook, Instagram y YouTube), entre estudiantes de secundaria (Generación Z) y universitarios (Generación $\mathrm{Y})$ ?

PI2 ¿Qué gratificaciones se asocian con la intensidad de uso de las redes sociales (Facebook, Instagram y YouTube) por los estudiantes de secundaria (Generación Z) y por los estudiantes universitarios (Generación Y)?

\section{Datos y método de la investigación}

\section{Recogida de datos y participantes}

La investigación llevada a cabo se estructura en torno a dos estudios transversales y transgeneracionales a partir de los cuáles recoger los datos necesarios para dar respuesta a las preguntas de investigación. En el estudio A participaron 260 adolescentes, con edades comprendidas entre los 13 y los 18 años, estudiantes de Institutos de Educación Secundaria de tres provincias españolas (Cantabria, Madrid y Huelva). En el estudio B participaron 315 jóvenes de entre 19 y 38 años, estudiantes en universidades públicas de las mismas provincias.

Para proceder a recoger los datos se llevó a cabo una selección de Institutos de Educación Secundaria y de universidades, de las tres provincias, de manera aleatoria, a partir de un listado oficial de los centros públicos disponibles en los datos abiertos de cada Consejería de Educación. A continuación se solicitó la participación de los estudiantes en los estudios, tramitando los pertinentes permisos tanto con los estudiantes como con los responsables de los centros, y en su caso, los responsables de los menores de edad, y comunicando cuáles eran los objetivos de la investigación, así como su implicación voluntaria y anónima en el proceso de recogida de datos.

\section{Medidas}

Medida de la intensidad de uso de redes sociales.

Cuando hablamos del uso de las redes sociales es habitual tratar de expresar o comprender este uso en función del tiempo que un usuario está conectado a estas redes, la frecuencia con la que las utilizan o la duración de uso de las mismas. Este enfoque parece limitado puesto que no atiende a la actividad que un usuario puede realizar en una red, ni a la riqueza de la experiencia que está viviendo, tal y como proponen Valenzuela, Park, \& Kee (2009). En esta investigación se ha tenido en cuenta la propuesta de Brinder Howes, \& Sutcliffe (2009), puesto que incluye la interacción en línea, es decir, atendiendo al tiempo que el usuario está usando la red y el número de conexiones que tienen en sus redes.

\section{Medida de las escalas de Usos y Gratificaciones.}

Para el diseño del instrumento de medida de Usos y Gratificaciones se definieron 18 ítems, correspondientes a cinco gratificaciones del uso de los distintos SRS, aplicándose una escala tipo Likert con valores que van desde "Totalmente en desacuerdo" (1) hasta "Totalmente de acuerdo" (5), que son: Evasión, Entretenimiento, Presencia social, Auto-presentación y Logro. La Teoría de usos y gratificaciones ofrece una amplia variedad de gratificaciones que motivan a los usuarios a utilizar unas u otras redes, tal y como se ha justificado previamente, sin embargo y teniendo en cuenta la muestra en la que esta investigación se centra, se han seleccionado estas cinco gratificaciones por ser las más directamente asociadas a los jóvenes, tal y como justifican los trabajos de Urista, Dong \& Day (2009).

Para realizar el análisis factorial confirmatorio de los 18 ítems del instrumento de medida de Usos y Gratificaciones se aplicó el algoritmo de Máxima Verosimilitud (ML), prescindiendo de un análisis factorial exploratorio, puesto que ya se había definido una estructura factorial previa. Por lo tanto, se formuló la hipótesis de que la medida de 18 ítems representaría la estructura de cinco factores, examinando los pesos de regresión de estandarización y los valores de correlación múltiple cuadrados para todos los ítems, tal y como puede observarse en la Tabla 1. Así, los valores recomendados para la bondad del ajuste del modelo son: $\mathrm{c}^{2} / \mathrm{df}<3$, índice de ajuste comparativo $(\mathrm{CFI}) \geq 0.92$, índice Tucker-Lewis $(\mathrm{TLI}) \geq 0.92$, y el error medio cuadrático de aproximación (RMSEA) $<0.08$

Los resultados obtenidos sugieren que tanto el Estudio A $\left(\mathrm{c}^{2} /\right.$ $\mathrm{df}=2,125, \mathrm{IFI}=0.90, \mathrm{CFI}=0.90, \mathrm{TLI}=0.86, \mathrm{RMSEA}=0.06)$, como el Estudio B $\left(c^{2} / \mathrm{df}=2.498, \mathrm{CFI}=0.90, \mathrm{TLI}=0.86, \mathrm{RMSEA}=0.06\right)$, poseen un ajuste al modelo satisfactorio.

Tabla 1. Análisis factorial confirmatorio utilizando tres estudios transversales

Variables

Evasión (EVA)

Uso Internet cuando me siento frustrado

Uso Internet cuando he tenido un mal día

Internet me permite sacar mi ira

Internet es la mejor manera de evadirme del mundo

Entretenimiento (ENT)

Uso Internet porque es entretenido

Porque me divierte probar aplicaciones nuevas

Porque me ayuda a relajarme

Para expresarme libremente
Estudio A (13-18 años) $(\mathrm{N}=260)$
Beta
$\mathrm{R}^{2}$

, 898

, 880

,633

,671

, 631

, 595

,789

, 802
, 807

,775

,400

, 450

,398

,354

,623

,643
Estudio B (19-38 años) ( $\mathrm{N}=315)$

Beta $\mathrm{R}^{2}$

,920

.846

,928

.861

,494

.244

.309

.465

.526

.583

,763

.420 
Variables

Para ocupar mi tiempo

Para superar el aburrimiento y la soledad

Presencia Social (PS)

Confío en que otras personas me ayudarán si lo necesito

Me siento conectado a otras personas

Soy capaz de ser yo mismo y mostrar que clase de persona soy

Me siento muy integrado en mis redes

Auto-presentación (AP)

En Internet quiero que otras personas me perciban como agradable

Quiero que otras personas me perciban como inteligente

Logro (L)

Uso Internet para tener más poder/influencia que otros

Uso Internet para demostrar a otros que soy el mejor

Respecto a la fiabilidad del instrumento se calculó el coeficiente Alpha de Cronbach y la confiabilidad compuesta (CR). La validez del instrumento se midió a través de la carga del factor y la varianza promedio (AVE). La validez discriminante a partir

$\begin{array}{cccc}\text { Estudio A (13-18 años) }(\mathrm{N}=260) & \text { Estudio B (19-38 años) }(\mathrm{N}=315) \\ \text { Beta } & \mathrm{R}^{2} & \text { Beta } & \mathrm{R}^{2} \\ , 732 & , 535 & , 734 & .539 \\ , 664 & , 441 & , 643 & .413 \\ & & & \\ , 703 & , 495 & , 598 & .357 \\ , 806 & , 649 & , 738 & .545 \\ , 648 & , 420 & , 572 & .327 \\ , 744 & , 553 & & \\ & & & .928 \\ , 926 & , 963 & .745 \\ , 895 & , 801 & , 863 & .867 \\ & , 857 & & .631 \\ , 695 & & , 931 & \end{array}$

del cálculo de las correlaciones entre los dos factores y su contraste con la raíz cuadrada de la varianza. Todos los valores resultantes respecto a la validez y a la fiabilidad del instrumento obtuvieron índices aceptables (Tabla 2).

Tabla 2. Análisis descriptivo y de la validez convergente y discriminante de los constructos utilizados en los estudios A y B

\begin{tabular}{lcrrrrrrr}
\multicolumn{1}{l}{ Estudio A (13-18 años) $(\mathrm{N}=260)$} & & & & & & & \\
& Media(SD) & CR & AVE & EVA & ENT & PS & AP & L \\
EVA $(\alpha=.84)$ & $2,11(0.88)$ & .89 & .68 & .82 & & & & \\
ENT $(\alpha=.85)$ &, $74(0.78)$ & .88 & .56 & .69 & .75 & & & \\
PS $(\alpha=.80)$ & $2,21(0.80)$ & .87 & .63 & .38 & .54 & .79 & & \\
AP $(\alpha=.90)$ & $2,25(1.07)$ & 94 & .90 & .30 & .34 & .51 & .95 & \\
L $(\mathrm{a}=.55)$ & $1,28(0.52)$ & .81 & .69 & .37 & .25 & .24 & .18 & .83 \\
Estudio B $(19-38$ años) $(\mathrm{N}=315)$ & & & & & & & \\
& Media(SD) & CR & AVE & EVA & ENT & PS & AP & L \\
EVA $(\alpha=.81)$ & $1.83(0.69)$ & .82 & .56 & .75 & & & & \\
ENT $(\alpha=.85)$ & $2.44(0.70)$ & .85 & .49 & .56 & .70 & & & \\
PS $(\alpha=.64)$ & $2.11(0.66)$ & .67 & .40 & .26 & .33 & .64 & & \\
AP $(\alpha=.90)$ & $2.16(1.00)$ & .91 & .83 & .24 & .35 & .51 & .91 & \\
L $(\alpha=.85)$ & $1.23(0.53)$ & .85 & .74 & .22 & .37 & .22 & .15 & .86
\end{tabular}

\section{Análisis de datos}

Para dar respuesta a la $\mathbf{P 1}$, se aplicó el test-t para muestras independientes.

Para responder a la $\mathbf{P 2}$ se aplicó un análisis de la regresión lineal en los estudios A (estudiantes de secundaria) y B (estudiantes universitarios), siendo la variable dependiente la intensidad de uso $\left(f^{*} n^{\underline{o}}\right.$ de conexiones), y las variables independientes las dimensiones: evasión, entretenimiento, presencia social, auto-presentación y logro. Los análisis de la regresión lineal se aplicaron a las tres redes sociales genéricas consideradas en el estudio.

\section{Análisis y Resultados}

Uso de las redes sociales por adolescentes y jóvenes: análisis comparativo

Para comprender las diferencias entre los estudiantes adolescentes y los jóvenes universitarios respecto al uso de las tres redes sociales objeto de estudio en la investigación se aplicó la prueba $t$ para muestras independientes. Los resultados (Tabla 3) indican que los adolescentes y los jóvenes universitarios difieren en la frecuencia de uso de Facebook $(t=-15.01, d f=562, p \leq 0.001)$, en el uso de Instagram $(t=2.02, d f=556, p \leq 0.05)$, y en el uso de YouTube $(t=3.59, d f=553, p \leq 0.001)$. A partir de esta prueba se constata que los estudiantes de secundaria utilizan con mayor frecuencia Instagram y YouTube [Media (SD) $=5,15(1,40)$ y 5,02 
$(1,28)]$, que los jóvenes universitarios [Media (SD) $=4,87(1,80)$ y $4,61(1,41)]$. Respecto a Facebook, los estudiantes universitarios lo utilizan con mayor frecuencia [Media (SD) $=4,64(1,67)$ ] que los estudiantes de secundaria [Media $(\mathrm{SD})=2,47(1,76)]$.

Tabla 3. Prueba $t$ para muestras independientes

$\begin{array}{llllll} & \text { Estudio A (13-18 años) } & \text { Estudio B (19-38 años) } & & & \\ & \text { Media(SD) } & \text { Media(SD) } & \text { t-valor } & d f & p \text {-valor } \\ \text { Facebook } & 2,47(1.76) & 4.64(1.67) & -15.01 & 562 & .000 \\ \text { Instagram } & 5.15(1.40) & 4.87(1.80) & 2.02 & 556 & .043 \\ \text { YouTube } & 5.02(1.28) & 4.61(1.41) & 3.59 & 553 & .000\end{array}$

Gratificaciones asociadas al uso intenso de las redes sociales

En esta investigación se ha tratado de conocer la gratificación que encuentran los estudiantes de secundaria y los universitarios en las redes sociales, relacionándolo con la frecuencia de uso. En la Tabla 4 se muestran los resultados de las regresiones lineales respecto a Facebook, Instagram y YouTube en los dos grupos o estudios.

Respecto a Facebook, la presencia social es la gratificación asociada significativamente con la frecuencia de uso, en el caso de los adolescentes $(\beta=.23, t$-value $=2.72, p$-value $=.007)$. Respecto a los jóvenes universitarios no se encontraron relaciones significativas.
Respecto a Instagram, también es la presencia social la gratificación asociada significativamente con la frecuencia de uso, en el caso de los adolescentes $(\beta=.23, t$-value $=2.72, p$-value $=.007)$. Tampoco se encontraron relaciones significativas en el caso de los jóvenes universitarios.

Los resultados obtenidos en YouTube presentan similares características, de manera que la presencia social fue nuevamente la gratificación asociada significativamente con la intensidad de uso para los estudiantes adolescentes $(\beta=.38, t$-value $=4.77, p$-value $=.000)$, mientras que para los estudiantes universitarios la gratificación asociada con la frecuencia de uso fue el entretenimiento $(\beta=.22, t$-valor $=2.93, p$-valor $=.004)$.

Tabla 4. Resultados de la regresión lineal*

\begin{tabular}{|c|c|c|c|c|c|c|}
\hline \multirow[b]{2}{*}{ Facebook UEG } & \multicolumn{2}{|c|}{ Estudio A (N=260) (13-18) } & \multicolumn{4}{|c|}{ Estudio B (N=315) (19-38) } \\
\hline & Beta & $t$-valor & $p$-valor & Beta & $t$-valor & $p$-valor \\
\hline Evasión & ,036 & 410 & ,682 & ,046 & ,624 &, 533 \\
\hline Entretenimiento &,- 014 &,- 159 & 874 & ,111 & 1,453 & ,147 \\
\hline Presencia Social & ,232 & 2,721 & 007 & ,062 & ,931 & ,352 \\
\hline Auto presentación &, 064 & 860 & ,391 & ,003 & 040 & ,968 \\
\hline Logro & 109 & 1,541 & ,125 &, 031 & 490 & ,624 \\
\hline$R^{2}$ valor & .08 & & & .02 & & \\
\hline \multicolumn{7}{|l|}{ Instagram UEG } \\
\hline Evasión & 040 & 489 & 625 & 044 & 600 &, 549 \\
\hline Entretenimiento &,- 003 &,- 038 & ,970 & ,136 & 1,775 & ,077 \\
\hline Presencia Social & ,382 & 4,776 & 000 & ,036 &, 531 &, 596 \\
\hline Auto presentación & 163 & 2,342 & ,020 &,- 009 &,- 130 & 897 \\
\hline Logro &,- 044 &,- 662 &, 509 & 077 & 1,200 & ,231 \\
\hline$R^{2}$ valor & .19 & & & .03 & & \\
\hline \multicolumn{7}{|l|}{ YouTube UEG } \\
\hline Evasión & ,045 &, 533 &, 595 & ,006 & ,084 & ,933 \\
\hline Entretenimiento & ,044 &, 524 & 601 & ,220 & 2,937 & ,004 \\
\hline Presencia Social & ,374 & 4,525 & ,000 &,- 012 &,- 178 & ,859 \\
\hline Auto presentación & ,094 & 1,310 & 192 & ,033 & ,483 & ,630 \\
\hline Logro &,- 018 &,- 271 & 787 &, 089 & 1,414 & 159 \\
\hline$R^{2}$ valor & .16 & & & .07 & & \\
\hline
\end{tabular}

*Variable dependiente: intensidad de uso (frecuencia * $\mathrm{n}^{\mathrm{o}}$ de conexiones) de Facebook, Instagram y YouTube.

\section{Discusión y conclusiones}

La utilización de las redes sociales como recurso educativo se viene implementando con éxito en algunas experiencias in- novadoras, especialmente vinculadas con el ámbito universitario y en menor medida con los estudios de secundaria (González, Lleixà \& Espuny, 2016; Hershkovizt y Forkosh-Baruch, 2017; Yeboah \& Ewur, 2014) Sin embargo, no hay suficientes evidencias 
científicas que permitan conocer cuáles son las motivaciones o las gratificaciones que los estudiantes encuentran para hacer uso de ellas fuera del contexto educativo en función de la frecuencia de uso. En este trabajo se ha pretendido conocer cuáles son las redes más utilizadas por parte de cada uno de estos dos grupos de estudiantes, así como cuáles son los motivos o gratificaciones que hacen que utilicen unas $u$ otras, evidenciando si son diferentes o no. Teniendo en cuenta los resultados obtenidos se pretende aportar algunas pautas al profesorado que mejoren significativamente el aprovechamiento de las redes sociales en las actividades vinculadas al ámbito académico, seleccionando aquellas más utilizadas por cada grupo de estudiantes y tomando en consideración cuáles son los motivos por los que las eligen.

A partir de las evidencias ofrecidas en este trabajo, el profesorado podrá seleccionar aquellas redes con las que los estudiantes se sienten más habituados a buscar información, compartirla, crear contenidos propios $u$ otras múltiples actividades que permite cada una de las redes analizadas en este estudio, Facebook, Instagram y YouTube. Podrán, en definitiva, apropiarse de las redes sociales para ofrecer a los estudiantes de secundaria y universitarios nuevas propuestas didácticas, cuyo sentido y significado no repercuta solo en el ámbito académico, sino que transcienda a su entorno digital, configurando un entorno virtual de aprendizaje que dote al estudiante de las herramientas necesarias para mejorar sus competencias, conocimientos, actitudes y destrezas.

El profesorado de secundaria tendrá la oportunidad de mejorar las prácticas de aprendizaje utilizado de una manera más eficaz Instagram y YouTube, puesto que respecto a la PI1 estas son las redes preferidas por esta generación, principalmente para conseguir tener más presencia social, buscando también la auto-presentación. Los resultados del estudio han mostrado también, en relación a la PI2, la especial preocupación de esta generación $Z$ por cuidar la imagen que transmiten en sus perfiles a través de sus redes. Por lo tanto, aquellas actividades o propuestas didácticas que ofrezcan la posibilidad de favorecer la presentación de una imagen favorable, dentro del ámbito académico y del aprendizaje, tiene mayores posibilidades de ser bien acogida por los estudiantes y de resultar mucho más significativas para su aprendizaje.

Por otro lado, a partir de los resultados del estudio con jóvenes universitarios hemos conocido que esta generación $\mathrm{Y}$ prefiere la utilización de Facebook como red social, respecto a la PI1. En cuanto a la PI2, los datos sugieren que las gratificaciones que buscaban estos jóvenes para usar intensamente esta red están relacionadas con el entretenimiento. A partir de estos datos podemos concluir que la utilización de Facebook en los estudios universitarios será más exitoso que utilizar otras redes sociales. Estos resultados coinciden con estudios como los de Igartua \& Rodríguez-de-Dios (2016), asociando el uso de esta red con la expresión libre y con momentos de ocio.

Finalmente es preciso insistir en la necesidad de seleccionar las redes sociales que se pueden utilizar en el contexto de aprendizaje atendiendo a la generación con la que se va a trabajar, tal y como se ha podido constatar en este estudio, más allá de tener en cuenta como premisa las características, las posibilidades o aplicabilidad de las diferentes redes sociales.

A partir de los resultados encontrados, teniendo en cuenta las limitaciones del estudio que podrían superarse teniendo en consideración otras gratificaciones, otras redes sociales, o llevando a cabo un estudio longitudinal, puede considerare que los hallazgos del estudio tienen importantes implicaciones teóricas y prácticas para la comunidad educativa, en tanto en cuanto, se ofrecen datos contrastados que constituyen una guía para seleccionar las redes sociales más adecuadas para trabajar con cada generación de estudiantes de una manera más eficaz, puesto que se han conocido cuáles son los motivos o gratificaciones por las que hacen un mayor uso de cada una de las redes estudiadas.

Este trabajo se ha elaborado en el marco de la "Red de Educación Mediática" del Programa Estatal de Investigación Científica-Técnica de Excelencia, Subprograma Estatal de Generación de Conocimiento (EDU2016-81772-REDT).

\section{Referencias bibliográficas}

Almansa-Martínez, A., Fonseca, O., \& Castillo-Esparcia, A. (2013). Redes sociales y jóvenes. Uso de Facebook en la juventud colombiana y española. Comunicar, 20(40), 127-135. https://doi.org/10.3916/C40-2013-03-03

Andújar-Vaca, A., \& Cruz-Martínez, M. (2017). Mobile Instant Messaging: Whatsapp and its Potential to Develop Oral Skills. Comunicar, 50, 43-52. https://doi.org/10.3916/C50-2017-04

Ayorech, Z., von Stumm, S., Haworth, C. M., Davis, O. S., \& Plomin, R. (2017). Personalized media: a genetically informative investigation of individual differences in online media use. PloS one, 12(1), https://doi.org/10.1371/journal. pone. 0168895

Barajas Meneses, F., \& Álvarez Morán, C. (2013). Uso de Facebook como herramienta en la enseñanza del área de naturales en el grado undécimo de educación media vocacional. PixelBit. Revista de Medios y Educación, (42), 143-156. Recuperado de: https://goo.gl/ep4wnQ

Binder, J., Howes, A., Sutcliffe, A. (2009). The problem of conflicting social spheres: effects of network structure on experienced tension in social network sites. In: Proceedings of the 27th International Conference on Human Factors in Computing System, New York, ACM, 965-974.

Burbules, N. C. (2016). How We Use and Are Used by Social Media in Education. Educational Theory, 66(4), 551-565. https:// doi.org/10.1111/edth.12188

Coombes, B. (2009). Generation Y: Are they really digital natives or more like digital refugees? Synergy, 7 (1), 31-40. Recuperado de: https://goo.gl/k36Kwq

Dumas, T. M., Maxwell-Smith, M., Davis, J. P., \& Giulietti, P. A. (2017). Lying or longing for likes? Narcissism, peer belonging, loneliness and normative versus deceptive like-seeking on Instagram in emerging adulthood. Computers in Human Behavior, 71, 1-10. https://doi.org/10.1016/j.chb.2017.01.037

Fernández-Cruz, F. J., \& Fernández-Díaz, M.J. (2016). Los docentes de la Generación Z y sus competencias digitales. Comunicar, 24(46), 97-105. https://doi.org/10.3916/C46-2016-10

Fondevila-Gascon, J.F., Mir, P., Aguinaga, P., Carreras, M., \& Del Olmo, J.L. (2014). Use of instant messaging among spanish Young population: uses and abuses. 7th International Conference of Education, Research and Innovation, Sevilla. Recuperado de: https://goo.gl/hw7y7Z

Fuentes, V., García, M., \& Aranda, M. (2017). Grupos de clase; grupos de whatsapp. Análisis de las dinámicas comunicativas entre estudiantes universitarios. Prisma Social, (18). Recuperado de: https://goo.gl/xH7WS8

García Galera, M. C., \& Fernández Muñoz, C. (2016). Si lo vives, lo compartes: Cómo se comunican los jóvenes en un mundo digital. Madrid: Fundación Telefónica. Recuperado de: https:// goo.gl/JPJuJ5

García-Ruiz, R., Ramírez, A., \& Rodríguez, M.M. (2014). Educación en alfabetización mediática para una nueva ciudadanía prosumidora. Comunicar, 43, 15-23. https://doi.org/10.3916/ C43-2014-01

Gathegi, J. N. (2014). Social media networking literacy: rebalancing sharing, privacy, and legal observance. In European 
Conference on Information Literacy, 101-108. Recuperado de: https://goo.gl/ESQInq

Gerják, I. (2016) Teaching digital image processing - Eyes and eyesight. Annales Mathematicae et Informaticae, (47), 229-242. Recuperado de: https://goo.gl/Vhrxws

Gonzales, A., \& Hancock, J. (2011). Mirror, mirror on my Facebook wall: effects of exposure to Facebook on self-esteem. Cyberpsychol. Behav. Soc. Netw. 14 (1-2), 79-83. https://doi. org/10.1089/cyber.2009.0411

Herrero-Diz, P., Ramos-Serrano, M., \& Nó, J. (2016). Los menores como usuarios creadores en la era digital: del prosumer al creador colaborativo. Revisión teórica 1972-2016. Revista Latina de Comunicación Social, (71), 1301-1322. http://dx.doi. org/10.4185/RLCS-2016-1147

Hershkovizt, A., \& Forkosh-Baruch, A. (2017). Teacher-Student Relationship and Facebook-Mediated Communication: Student Perceptions. Comunicar, 53, 91-101. https://doi. org/10.3916/C53-2017-09

Howe, N., \& Strauss, W. (2000). Generations, the History of America's Future, 1584-2069. Nueva York: HarperCollins.

Hu, Y., Manikonda, L., \& Kambhampati, S. (2014). What We Instagram: A First Analysis of Instagram Photo Content and User Types. Proceedings of the Eighth International AAAI Conference on Weblogs and Social Media, 595-598. Recuperado de: https://goo.gl/yycxcz

Igartua, J., \& Rodríguez-de-Dios, I. (2016). Correlatos motivacionales del uso y la satisfacción con Facebook en jóvenes españoles. Cuadernos.Info, (38), 107-119. https://doi.org/10.7764/ cdi. 38.848

Izrael, P. (2017). To be on facebook or not to be: slovak young people and their use of social networking sites. Informatologia, 50(1-2), 63-73.

Jordán-Conde, A., Mennecke, B., \& Townsend, A. (2014). Late adolescent identity definition and intimate disclosure on Facebook. Computers in Human Behavior, 33, 356-366. https://doi. org/10.1016/j.chb.2013.07.015

Junco, R., (2012). Too much face and not enough books: the relationship between multiple indices of Facebook use and academic performance. Comput. Hum.Behav. 28(1), 187-198. https://doi.org/10.1016/j.chb.2011.08.026

Katz, E., Blumer, J.G., \& Gurevitch, M. (1973). Uses and gratifications research. The Public Opinion Quarterly, 37(4), 509-523. Recuperado de: https://goo.gl/S7Ekwg

Leung, L. (2013). Generational differences in content generation in social media: The roles of the gratifications sought and of narcissism. Computers in Human Behavior, 29(3), 997-1006. http://dx.doi.org/10.1016/j.chb.2012.12.028

Manzi, C., Coen, S., Regalia, C., Yevenes, A. M., Giuliani, C., \& Vignoles, V. L. (2018). Being in the Social: A cross-cultural and cross-generational study on identity processes related to Facebook use. Computers in Human Behavior, 80, 81-87. https:// doi.org/10.1016/i.chb.2017.10.046

Marsh, J. (2014). The relationship between online and offline play: Friendship and exclusion. En A. Burn y C. Richards (eds.) Children's games in the new media age: Childlore, media and the playground. (pp. 109-132) London: Ashgate. Recuperado de: https://goo.gl/TCJo66

González, J. G., Lleixà, M., \& Espuny, C. (2016). Las redes sociales y la educación superior: las actitudes de los estudiantes universitarios hacia el uso educativo de las redes sociales, de nuevo a examen. Education in the Knowledge Society, 17(2), 2138. http://dx.doi.org/10.14201/eks20161722138

Oberts, Ú., Chamarro, A., \& Renau, V. (2016). Estereotipos de género 2.0_Auto-representaciones de adolescents en Facebook. Comunicar, 48, 81-90. https://doi.org/10.3916/C48-2016-08
Palmgreen, P., \& Rayburn, J. D. (1979). Uses and gratifications and exposure to public television: A discrepancy approach. Communication Research, 6(2), 155-179. https://doi. org $/ 10.1177 / 009365027900600203$

Parra Castrillón, E. (2010). Las redes sociales de Internet: también dentro de los hábitos de los estudiantes universitarios. Anagramas Rumbos y Sentidos de la Comunicación, 9(17), 107-116. Recuperado de: https://goo.gl/huyQFi

Pérez-Escoda, A. (2016). La competencia digital de la Generación Z: claves para su introducción curricular en la Educación Primaria. Comunicar, 24(49), 71-80. https://doi.org/10.3916/C49$\underline{\text { 2016-07 }}$

Rubio-Romero, J., \& Perlado Lamo de Espinosa, M. (2015). El fenómeno WhatsApp en el contexto de la comunicación personal: una aproximación a través de los jóvenes universitarios, Icono 14, 13(4), 73-94. https://doi.org/10.7195/ri14.v13i2.818

Sabater, I., Martínez, R., \& Santiago, R. (2017) La Tecnosocialidad: El papel de las TIC en las relaciones sociales. Revista Latina de Comunicación Social, 72, 1592 -1607. https://doi. org/10.4185/RLCS-2017-1236

Schroer, W. J. (2008). Defining, Managing, and Marketing to Generations X, Y, and Z. The Portal, 10, 9, 9-10 Recuperado de: http://goo.gl/Fc40dB

Smith, A., Fischer, E., \& Yongjian, C. (2012). How Does Brand-related User-generated Content Differ across YouTube, Facebook, and Twitter? Journal of Interactive Marketing, 26(2), 102113. https://doi.org/10.1016/j.intmar.2012.01.002

Svensson, J., \& Russmann, U. (2017). Introduction to visual communication in the age of social media: Conceptual, theoretical and methodological challenges. Media and Communication, 5(4), 1-5. http://dx.doi.org/10.17645/mac.v5i4.1263

Tur, G., Marín-Juarros, V., \& Carpenter, J. (2017). Using Twitter in Higher Education in Spain and the USA. Comunicar, 51, 19-28. https://doi.org/10.3916/C51-2017-02

Urista, M.A., Dong, Q., \& Day, K.D. (2009). Explaining why young adults use myspace and facebook through uses and gratifications theory. Human Communication, 12(2), 215- 229. Recuperado de: https://goo.gl/f8pPHu

Valenzuela, S., Park, N., \& Kee, K.F. (2009). Is there social capital in a social network site? Facebook use, and college students' life satisfaction, trust, and participation. J. Comput.-Mediated Commun. 14(4), 875-901.

Vasanth R., \& Swamy, S. (2013). Social Media's Impact on Teenagers. International Conference on Cross-Cultural Design, 477485. https://doi.org/10.1007/978-3-642-39143-9 53

Wilson, R. E., Gosling, S. D., \& Graham, L. T. (2012). A review of Facebook research in the social sciences. Perspectives on psychological science, 7(3), 203-220. https://doi. org/10.1177/1745691612442904

Xie, W. (2014) Social network site use, mobile personal talk and social capital among teenagers. Computers in Human Behavior, 41, 228-235. https://doi.org/10.1016/j.chb.2014.09.042

Yarosh, S., Bonsignore, E., McRoberts, S., \& Peyton, T. (2016). YouthTube: Youth video authorship on YouTube and Vine. In Proceedings of the 19th ACM Conference on Computer-Supported Cooperative Work E Social Computing, 1423-1437. http://dx. doi.org/10.1145/2818048.2819961

Yeboah, J., \& Ewur, D. (2014). The Impact of WhatsApp Messenger Usage on Students Performance in Tertiary Institutions in Ghana. Journal of Education and Practice, 5(6), 157- 164- Recuperado de: https://goo.gl/qr9KoP

Zhitomirsky-Geffet, M., \& Blau, M. (2016). Cross-generational analysis of predictive factors of addictive behavior in smartphone usage. Computers in Human Behavior, 64, 682-693. https://doi.org/10.1016/j.chb.2016.07.061 